\title{
Distribuição espacial da COVID-19 no estado da Paraíba: uma associação com a densidade demográfica
}

\author{
COVID - 19 spatial distribution in the state of Paraíba: an association with demographic density \\ COVID - 19 distribución espacial en el estado de Paraíba: una asociación con densidad demográfica
}

\section{RESUMO}

Objetivo: Analisar e descrever a distribuição espacial dos dados epidemiológicos dos casos da COVID-19 no Estado da Paraíba. Método: Foi realizado um estudo ecológico descritivo, com base nas informações da COVID-19 na Paraíba, de acesso aberto do dashboard da Secretaria Estadual de Saúde, através dos dados de casos e óbitos confirmados. Após análise dos dados obtidos, foram transformados em taxas, a fim de compará-las com a densidade demográfica da região estudada. Resultados: Dentre os 223 municípios do Estado da Paraíba, aqueles com uma maior densidade demográfica lideraram as taxas de morbidade, incidência e letalidade da COVID-19 no Estado, durante o período estudado. Conclusão: Pôde-se observar a contribuição das grandes cidades como fonte de propagação da doença, por sua alta densidade demográfica, reafirmando assim, a necessidade e a importância das medidas preventivas de saúde pública a serem adotadas, a fim de garantir um baixo nível de disseminação do vírus.
\end{abstract}

DESCRITORES: Infecções por Coronavírus; Densidade Demográfica; Epidemiologia descritiva.

\section{ABSTRACT}

Objective: To analyze and describe the spatial distribution of epidemiological data from COVID-19 cases in the state of Paraíba. Method: A descriptive ecological study was carried out, based on information from COVID-19 in Paraíba, with open access from the State Health Secretariat's dashboard, using confirmed case and death data. After analyzing the data obtained, they were transformed into rates, in order to compare them with the demographic density of the studied region. Results: Among the 223 municipalities in the State of Paraíba, those with a higher demographic density led the COVID-19 morbidity, incidence and lethality rates in the State, during the period studied. Conclusion: It was possible to observe the contribution of large cities as a source of the spread of the disease, due to its high demographic density, thus reaffirming the need and importance of preventive public health measures to be adopted, in order to guarantee a low level of spread of the virus.

DESCRIPTORS: Coronavirus Infections; Population Density; Epidemiology Descriptive.

\section{RESUMEN}

Objetivo: Analizar y describir la distribución espacial de datos epidemiológicos de casos de COVID-19 en el estado de Paraíba. Método: Se realizó un estudio ecológico descriptivo, con base en información del COVID-19 en Paraíba, con acceso abierto desde el tablero de la Secretaría de Salud del estado, utilizando datos de casos confirmados y defunciones. Luego de analizar los datos obtenidos, se transformaron en tasas, con el fin de compararlas con la densidad demográfica de la región estudiada. Resultados: Entre los 223 municipios del estado de Paraíba, los de mayor densidad demográfica lideraron las tasas de morbilidad, incidencia y letalidad del COVID-19 en el estado, durante el período estudiado. Conclusión: se pudo observar el aporte de las grandes ciudades como foco de propagación de la enfermedad, debido a su alta densidad demográfica, reafirmando así la necesidad e importancia de adoptar medidas preventivas de salud pública, a fin de garantizar un bajo nivel de propagación del virus.

DESCRIPTORES: Infecciones por Coronavirus; Densidad de Población; Epidemiología descriptiva.

RECEBIDO EM: 21/07/2020 APROVADO EM: 31/08/2020

\section{Lara Kamylli Felismino Chagas}

Presidente LASCEN. Graduanda do Curso de Enfermagem UNIFACISA.

ORCID: 0000-0002-7505-5036 


\section{Lúcia Magnólia Albuquerque Soares de Camargo}

Professora do Curso de Enfermagem, UNIFACISA. Coordenadora da Liga Acadêmica de Saúde Coletiva de Enfermagem - LASCEN. Mestre em Saúde Coletiva. Especialista em Gestão de Serviços de Saúde e Adm. Hospitalar, Saúde do Trabalhador e Saúde Pública. Enfermeira.

ORCID: 0000-0002-5442-8227

\section{Larissa Thays Brito Soares}

Membro LASCEN. Graduanda do Curso de Enfermagem UNIFACISA. ORCID: 0000-0003-0497-0752

\section{Letícia Ferreira Lemos}

Membro LASCEN. Graduanda do Curso de Enfermagem UNIFACISA. ORCID: 0000-0002-8218-6050

Maria Eduarda Carvalho Dias

Membro LASCEN. Graduanda do Curso de Enfermagem UNIFACISA. ORCID: 0000-0002-5119-180X

\section{Raiane Araújo Costa}

Membro LASCEN. Graduanda do Curso de Enfermagem UNIFACISA. ORCID: 0000-0001-7755-5862

\section{INTRODUÇÃO}

0 coronavírus vem de uma família de vírus que causam infecções respiratórias. $\mathrm{O}$ novo agente do coronavírus, no qual provoca a doença COVID-19, foi descoberto em 31 de dezembro de 2019 após casos registrados em Wuhan, na China ${ }^{(1)}$.

A COVID-19 é uma doença causada pelo coronavírus SARS-CoV-2, sua transmissão se dá a partir do contato próximo de uma pessoa contaminada com outra, através de gotículas de saliva, aperto de mão, espirro, tosse, secreções e por meio de objetos ou superfícies contaminadas. Os sintomas podem variar de um simples resfriado a uma pneumonia severa, sendo os mais comuns: tosse, dor de garganta, febre, coriza e dificuldade para respirar ${ }^{(1)}$.

No Brasil, o primeiro caso da COVID-19 foi confirmado quase dois meses depois do primeiro caso registrado no mundo, ocorrendo no dia 26 de fevereiro de 2020. O primeiro óbito confirmado ocorreu apenas em 17 de março de 2020. Após esse primeiro caso, dados já apontavam a tendência de que a doença tomaria grandes proporções rapidamente. Apenas um mês depois, já havia transmissão comunitária em algumas cidades do país. Com o Estado de São Paulo liderando o número de casos no Brasil, em março de 2020 foi reconhecida a transmissão comunitária da COVID-19 em todo o território nacional ${ }^{(2)}$.

O Estado da Paraíba, localizado no nordeste brasileiro, teve o primeiro caso confirmado da COVID-19 apenas no dia 21 de março de 2020. Tratava-se de um homem de 60 anos, morador da capital, João Pessoa/PB, com relato de uma viagem recente para Europa. O mesmo foi atendido em um hospital privado e esteve em isolamento domiciliar durante seu tratamento ${ }^{(3)}$.

A metodologia da análise espacial na saúde coletiva é utilizada, principalmente para avaliar e monitorar o ambiente para ser aplicado ao planejamento e avaliação dos serviços de saúde. Na situação atual de pandemia, a análise espacial é uma ferramenta importante, pois permite identificar áreas com maior propagação de doenças, consequentemente de maior risco, que permite direcionar melhor as políticas públicas de saúde ${ }^{(4)}$.

Ainda não há registros publicados de análise e descrição dos casos da COVID-19 no Estado da Paraíba de acordo com a Densidade Demográfica (DM), dessa forma, compreender a distribuição espacial desses casos é fundamental para desenvolver melhores políticas públicas nos estágios iniciais de surtos da COVID-19.

O objetivo deste trabalho foi analisar e descrever a distribuição espacial dos dados epidemiológicos dos casos da COVID-19 no Estado da Paraíba.

\section{MÉTODO}

Foi realizado um estudo ecológico descritivo, com base nas informações epidemiológicas da COVID-19 no Estado da Paraíba. Foram utilizados todos os dados de casos e óbitos confirmados de SARS- CoV-2 de acesso aberto do dashboard da Secretaria Estadual de Saúde da Paraíba. Como critério de inclusão foi definido o uso de dados das sete maiores cidades do Estado da Paraíba, a capital João Pessoa/ $\mathrm{PB}$, precedido de Campina Grande/PB, Guarabira/PB, Cabedelo/PB, Santa Rita/ $\mathrm{PB}$, Patos/PB e Mamanguape/PB.

O Estado da Paraíba possui uma população de aproximadamente $4.018 .127 \mathrm{mi}-$ lhões de habitantes, divididos entre os 223 municípios, segundo a estimativa 2019 do Instituto Brasileiro de Geografia e Estatística (IBGE), numa área de 56.467,242 $\mathrm{km} 2$, sendo a $13^{\mathrm{a}}$ região mais populosa do território brasileiro.

A Paraíba possui uma DM de 66,70 habitantes por $\mathrm{km} 2$. Também chamada de 
População Relativa, a DM leva em consideração a distribuição populacional em determinadas áreas, permitindo a identificação dos territórios mais e menos ocupados. O nordeste brasileiro possui a maior densidade entre as regiões no País, entre os estados desta região a Paraíba ocupa o quarto lugar no país ${ }^{(5)}$.

A população do estudo incluiu todos os casos - acumulados e novos, e os óbitos confirmados da COVID-19 nos municípios paraibanos, entre 21 de março, data do primeiro caso confirmado, e $01 \mathrm{de} \mathrm{Ju}$ lho de 2020.

As variáveis analisadas foram número de casos confirmados e número de óbitos. A pesquisa foi realizada com os dados registrados no período de 21 de março, dia do primeiro caso confirmado no Estado, a 01 de Julho de 2020, extraídos do Painel Coronavírus do Estado da Paraíba, que apresenta o monitoramento on-line dos casos da doença no país, informando a ocorrência de casos acumulados, novos e óbitos por região, Município, dia e semana epidemiológica.

Para compor os resultados obtidos, a partir do número absoluto de casos acumulados, novos e os óbitos confirmados pela COVID-19, foram calculadas taxas de incidência (TI), morbidade (TM), e letalidade (TL). Estas foram processadas com uso do programa Excel for Windows 365. Após análise dos dados obtidos através das taxas, foi realizado uma comparação desses resultados com a DM da região estudada.

Também foi realizada uma análise espacial, através do geoprocessamento de imagens foram extraídos do Laboratório de Estatísticas Aplicada ao Processamento de Imagens e Geoprocessamento (LEAPIG) da Universidade Federal da Paraíba (UFPB).

Não foi necessário submeter o estudo para aprovação do Comitê de Ética em Pesquisa, visto os dados serem de domínio público.

\section{RESULTADOS}

\section{Dos Casos Confirmados}

$\mathrm{Na}$ extensão territorial do Estado da Paraíba, há 223 municípios, dos quais 218, até o dia 01 de Julho de 2020 apresentaram casos confirmados para COVID-19. Observou-se certa proporcionalidade entre os municípios paraibanos que possuem uma maior DM, com uma maior taxa de casos confirmados pela COVID-19 no período estudado, ou seja as cidades de João Pessoa/PB, Campina Grande/PB, Guarabira/PB, Cabedelo/PB, Santa Rita/PB, Patos/PB e Mamanguape/PB. Da data de confirmação do primeiro caso até o período proposto para este estudo, foram registrados no Estado mais de 48 mil casos da doença, o que chegou a representar um TM de $1,1 \%$, ou seja, uma marca superior de mais de 1.190 casos da COVID-19, a cada 100 mil habitantes no Estado da Paraíba ${ }^{(5)}$, o que pode ser observado no quadro 1 a seguir.

Também pode ser observado no quadro 1 , que a capital paraibana, João Pessoa/PB, até o dia 01 de Julho de 2020, chegou a apresentar uma TM de $1,6 \%$ para, ou seja, 1.680 casos confirmados da COVID-19 a cada 100 mil habitantes no território. De acordo com o último censo demográfico do IBGE estima-se que a população da capital seja de aproximadamente 809 mil habitantes, resultando na maior do DM do Estado da Paraíba, $3.421,28 \mathrm{hab} / \mathrm{km}^{2(6)}$.

Em seguida, observa-se a cidade de

\section{Quadro 1: Taxa de Morbidade da COVID-19 na Paraíba até 01 de Julho de 2020 e} Densidade Demográfica

\begin{tabular}{|l|c|c|}
\hline \multicolumn{3}{|c|}{$\begin{array}{c}\text { TAXA DE MORBIDADE E DENSIDADE DEMOGRÁFICA DA } \\
\text { COVID-19 NA PARAIIBA - 2020 }\end{array}$} \\
\hline LOCAL & TAXA DE MORBIDADE (TM) & DENSIDADE DEMOGRÁFICA (DM) \\
\hline Paraîba & $1,1 \%$ & 66,7 \\
\hline João Pessoa/PB & $1,6 \%$ & $3.421,28$ \\
\hline Campina Grande/PB & $1,6 \%$ & 648,31 \\
\hline Guarabira/PB & $3,8 \%$ & 333,8 \\
\hline Cabedelo/PB & $2,7 \%$ & $1,815,57$ \\
\hline Santa Rita/PB & $0,8 \%$ & 165,52 \\
\hline Patos/PB & $1,30 \%$ & 212,82 \\
\hline Mamanguape/PB & $2,70 \%$ & 124,23 \\
\hline (Fonte: SES/PB, 2020) & \multicolumn{2}{|}{} \\
\hline
\end{tabular}

Campina Grande/PB, a qual até o dia 01 de Julho de 2020 apresentou uma TM de $1,6 \%$, para os casos da doença. A cidade possui uma DM de $648,31 \mathrm{hab} / \mathrm{km}^{2}$, sendo a segunda maior do $\operatorname{Estado}^{(7)}$.

Também é possível observar no quadro 1, dados do município de Guarabira/PB, o qual apresentou um dos primeiros casos confirmados no Estado, em 23 de abril de 2020, e e atingiu, até 01 de Julho de 2020 uma TM de 3,8\%, para uma cidade com DM de $333,80 \mathrm{hab} / \mathrm{km}^{2(8)}$.

Ocupando o quarto lugar no ranking de contaminação na Paraíba, observa-se no quadro 1 o município de Cabedelo/ PB, PB o qual apresentou seu primeiro caso de contaminação pela COVID-19 oito dias após o primeiro caso ser registrado na Paraíba, ocorrido no dia 9 de abril de 2020. O município é uma cidade portuária, possui uma DM de 1.815,57 e revelou uma TM de 2,7\% durante o período estudado $^{(9)}$.

O município de Santa Rita/PB, o quinto lugar em número de casos confirmados da COVID-19 na Paraíba, confirmou seus dois casos confirmados no dia 8 de abril de 2020, apenas sete dias após o primeiro caso registrado no Estado. Com uma DM de 165,52 hab/km2, durante o período de estudo o município de Santa Rita/PB apresentou uma TM de $0,8 \%$, de um total aproximadamente $1.500 \mathrm{ca}$ sos acumulados ${ }^{(10)}$. 


\section{artigo}

Ainda no quadro 1, observa-se que o sexto lugar em número de casos da $\mathrm{CO}$ VID-19 na Paraíba, até 01 de Julho de 2020, foi ocupado pelo município de Patos/PB, o qual apresentou três casos confirmados no dia 10 de abril de 2020. O Referido município possui uma DM de $212,82 \mathrm{hab} / \mathrm{km}^{2}$ e apresentou uma TM de $1,3 \%{ }^{(11)}$.

$\mathrm{O}$ município de Mamanguape/PB encontrou-se em sétimo lugar no ranking de contaminação no Estado da Paraíba, durante o período estudado. Apresentou seus dois primeiros casos confirmados apenas no dia 05 de maio de 2020, mas, em pouco mais de 20 dias chegou a marca de mais de 1.200 casos acumulados, representando uma TM de 2,7\%. O município possui uma DM de relativamente baixa, $124,23 \mathrm{hab} / \mathrm{km}^{2}$, porém é um importante polo agrícola do Estado, sendo uma região de grande tráfego ${ }^{(12)}$.
FIGURA 1: Distribuição Espacial de Casos Confirmados da COVID-19 na Paraíba até 01 de Julho de 2020

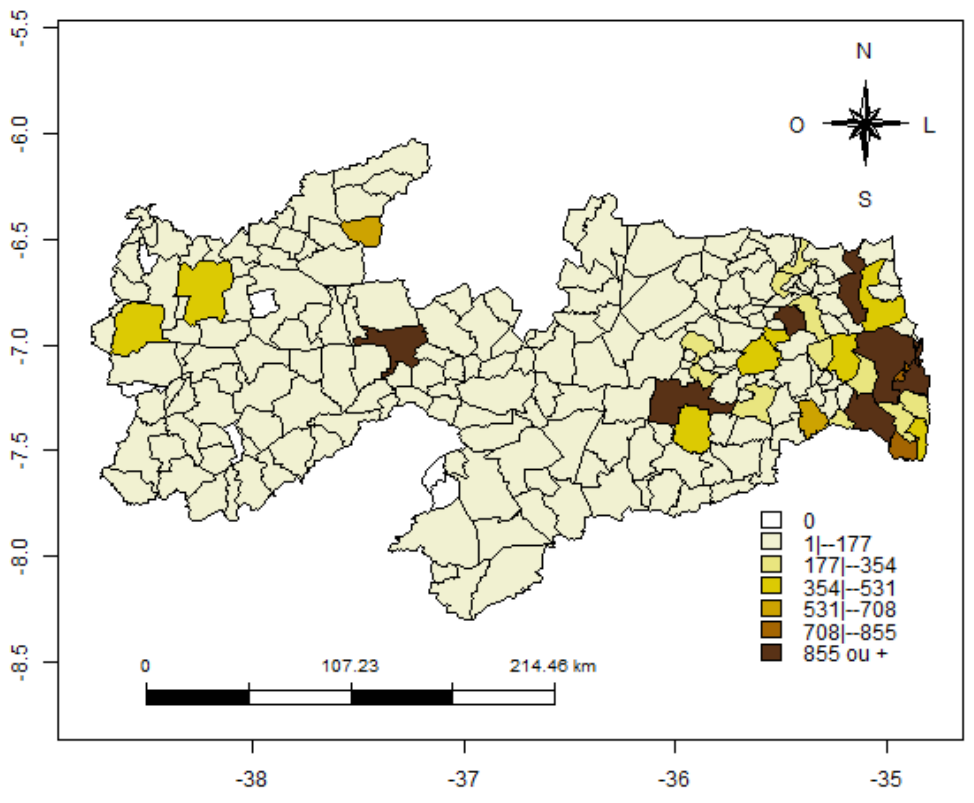

(FONTE: LEAPIG, 2020.)

Quadro 2: Taxa de incidência da COVID-19 na Paraíba até 01 de Julho de 2020

TAXA DE INCIDÊNCIA DA COVID-19 NA PARAÍBA - 2020

\begin{tabular}{|l|c|}
\hline LOCAL & TAXA DE INCIDÊNCIA (TI) \\
\hline Paraíba & $30,3 \%$ \\
\hline João Pessoa/PB & $35,8 \%$ \\
\hline Campina Grande/PB & $11 \%$ \\
\hline Guarabira/PB & $105,3 \%$ \\
\hline Cabedelo/PB & $48,71 \%$ \\
\hline Santa Rita/PB & $0 \%$ \\
\hline Patos/PB & $0 \%$ \\
\hline Mamanguape/PB & $127 \%$ \\
\hline (Fonte:SES/PB, 2020) & \\
\hline
\end{tabular}

Além da correlação da TM com a DM, também é possível notar, através distribuição espacial dos casos confirmados da COVID-19, desde o primeiro dia de caso confirmado no Estado até o dia 01 de Julho de 2020, conforme descrito acima, um processo de interiorização da COVID-19, tendo em vista que foi estabelecido um ranking de contaminação no Estado da Paraíba, no qual identifica-se esta tendência, de aumento da TM nas cidades mais interioranas, após disseminação nas maiores cidades, ou das cidades no entorno das grandes cidades do Estado, conforme ilustra a Figura 1, a seguir:

\section{Dos Novos Casos}

Diariamente, durante o período do estudo, foram registrados novos casos confirmados da COVID-19 no Estado da Paraíba, na grande maioria dos municípios deste Estado, o que possibilitou o direcionamento das políticas públicas de saúde, para nortear o Estado e o país, sobre a liberação e flexibilização das decisões sobre a pandemia. Estes novos casos confirmados da COVID-19 no Estado da Paraíba, podem ser observados, juntamente a TI, no quadro 2 a seguir.

No que se refere aos municípios que entraram como objeto neste estudo, até o dia 01 de Julho de 2020 foram registrados casos novos para COVID-19 apenas nos municípios de João Pessoa/PB, Campina Grande/PB, Guarabira/PB, Cabedelo/PB e Mamanguape/PB. Nos municípios de Santa Rita/PB e Patos/PB, nenhum caso novo da COVID-19 foi registrado, apresentando as primeiras tendências de queda no Estado da Paraíba.

Assim como apresentado no total de casos acumulados confirmados da COVID-19 no Estado da Paraíba, também é possível observar, através distribuição espacial dos novos casos confirmados durante o período proposto par ao estudo, mais uma vez o processo de interiorização da COVID-19, tendo em vista que foi estabelecido um ranking de contaminação no Estado da Paraíba, no qual identifica-se esta tendência, de aumento da TI nas cidades mais interioranas, após dissemi- 
nação nas maiores cidades, ou das cidades no entorno das grandes cidades do Estado conforme ilustra a Figura 2, a seguir:

\section{Dos Óbitos}

Entre 21 de março de 2020 e 01 de Julho de 2020 foram registrados no Estado da Paraíba mais de 1.00 óbitos confirmados pela COVID-19, o que chegou a totalizar 25 óbitos acumulados a cada 100 mil habitantes, representando uma TL de
2,08\% em todo o Estado, conforme apresenta o quadro 3 a seguir:

A maior TL no Estado da Paraíba, durante o período estudado, foi observado no município de Santa Rita/PB, com 6,70\%, enquanto a menor taxa no município de Guarabira/PB que chegou a registrar uma TM de $0,90 \%$. As cidades de João Pessoa/ PB e Campina Grande/PB tiveram uma TL mediana, o que pode ser associada ao acesso da população a rede de diagnóstico
FIGURA 2: Distribuição Espacial de Novos Casos da COVID-19 na Paraíba até 01 de Julho de 2020

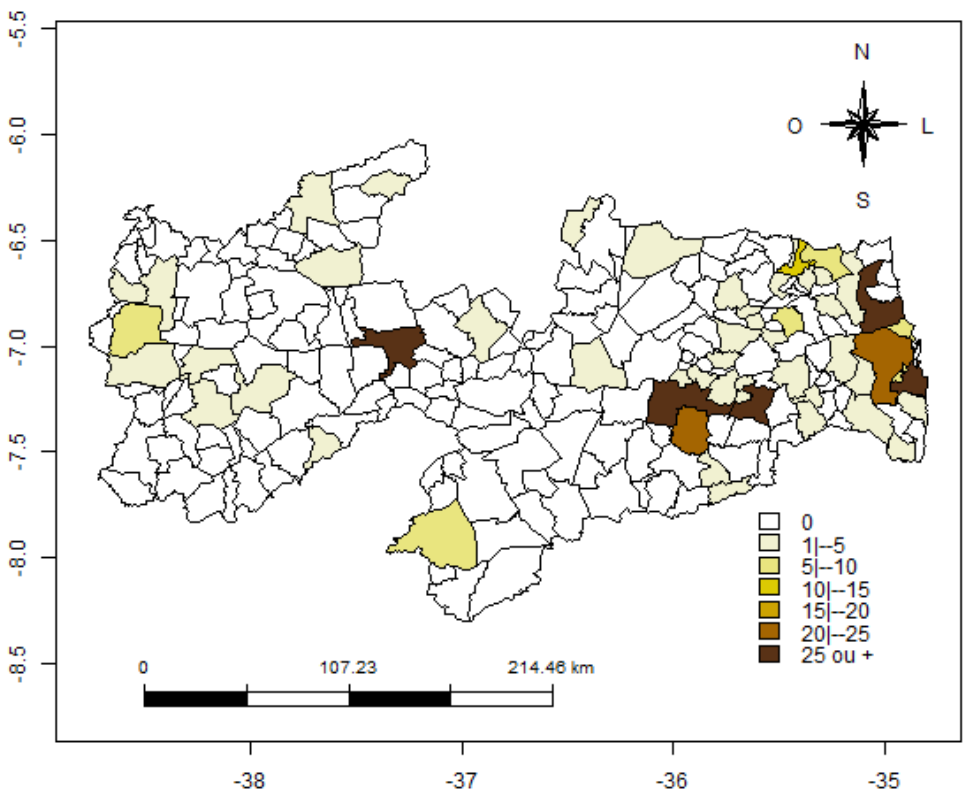

Fonte: LEAPIG, 2020.

Quadro 3: Taxa de Letalidade da COVID-19 na Paraiba até 01 de Julho de 2020

\begin{tabular}{|l|c|}
\hline \multicolumn{2}{|c|}{ TAXA DE LETALIDADE DA COVID-19 NA PARAÍBA - 2020 } \\
\hline LOCAL & TAXA DE LETALIDADE (TL) \\
\hline Paraíba & $2,08 \%$ \\
\hline João Pessoa/PB & $2,70 \%$ \\
\hline Campina Grande/PB & $1,70 \%$ \\
\hline Guarabira/PB & $0,90 \%$ \\
\hline Cabedelo/PB & $1,00 \%$ \\
\hline Santa Rita/PB & $6,70 \%$ \\
\hline Patos/PB & $3,80 \%$ \\
\hline Mamanguape/PB & $1,30 \%$ \\
\hline (Fonte: SES/PB, 2020) & \\
\hline
\end{tabular}

para a COVID-19, bem como internação e tratamento de alta complexidade.

Não possível o acesso a análise da distribuição espacial dos óbitos confirmados pela COVID-19 no Estado da Paraíba, durante o período proposto para este estudo, porém pode-se perceber a mesma tendência de aumento da TL nas cidades mais interioranas, após disseminação nas maiores cidades, ou das cidades no entorno das grandes cidades do Estado conforme o quadro 3 .

Dos óbitos confirmados pela CIVID-19 no Estado da Paraíba, durante o período estudado, aproximadamente $40 \%$ eram mulheres, mas a maioria de $60 \%$ eram homens. $30 \%$ das vítimas fatais da COVI-19 no Estado tinham idade maior ou igual a 80 anos, $21,5 \%$ estavam na faixa de idade entre 70 e 79 anos e 19\% tinham idade entre 60 e 69 anos, ou seja, quase $70 \%$ dos óbitos ocorreram entre pessoas de idade superior a 60 anos de idade. Grande parte das pessoas que vieram a óbito tinha alguma doença preexistente, dentre as principais, hipertensão, diabetes mellitus, cardiopatias e obesidade.

\section{DISCUSSÃO}

Tomando como base as DM semelhantes no território nacional, e a partir da avaliação da distribuição espacial dos dados epidemiológicos da COVID-19, através da TM, TI e TL no Estado da Paraíba, entre o primeiro caso diagnosticado até 01 de Julho de 2020, é possível comparar com os Estados do Ceará, de Santa Catarina, e do Rio Grande do Norte. A DM, bem como as TM, TI e TL dos Estados da Paraíba em comparação com o Ceará, Santa Catarina e Rio Grande no Norte, podem ser observados no quadro 4,5 e 6 a seguir.

No Quadro 4, observa-se uma correlação entre os dados da Paraíba e Santa Catarina, através dos valores de DM das sete maiores cidades do Estado de Santa Catarina, sendo João Pessoa/PB x Florianópolis/SC, por serem capitais, Campina Grande/PB x Joinville/SC, com média de DM 552 hab/km2, Guarabira/ $\mathrm{PB} \times$ Blumenau/SC, com média de DM 


\section{artigo}

466 hab/km2, Cabedelo/PB x São José/ SC, com média de DM 1.595 hab $/ \mathrm{km} 2$, Santa Rita/PB x Chapecó/SC com DM de $311 \mathrm{hab} / \mathrm{km} 2$ mediana. Patos/PB, Mamanguape/PB, Itajaí/SC e Criciúma/ $S C$, não revelaram similaridades entre suas $\operatorname{DM}^{(13,14,15,16,17,18,19,20)}$.

O Estado de Santa Catarina alcançou uma TM da COVID-19 de 0,3\%, durante o período proposto para o estudo, uma taxa bem menor quando comparado com a TM de 1,1\% alcançado no Estado da Paraíba no mesmo período. Continuando a análise, nas sete maiores cidades do Estado catarinense todas apresentaram TM menor em comparação as sete maiores cidades do Estado da Paraíba. Porém, a maior
TM catarinense ocorrida em Chapecó/ SC de $1,10 \%$, ultrapassou a TM de Santa Rita/PB, levando-se em consideração a semelhança entre a DM destes municípios, sendo o única ocorrência neste sentido.

Os demais municípios da Paraíba, incluídos neste estudo, apresentaram porcentagens com máxima de TM da COVID-19 de 3,8\% e mínima de $0,8 \%$, com média de 2,0\%, apresentando valores acima de Santa Catarina e da taxa apresentada pela federação nacional.

No que se refere a TI da COVID-19 no Estado da Paraíba, a comparação foi realizada com o Estado do Rio Grande do Norte, mais uma vez, em virtude da proporcionalidade da DM, porém as taxas apresentaram valores bastante divergentes, como apresenta-se no Quadro 5. Foram confrontadas as sete maiores cidades dos Estados da Paraíba e Rio Grande do Norte, comparando-se sua DM e a TI da COVID-19 no período proposto para o estudo. Observa-se que mais uma vez, o Estado da Paraíba, revela uma TI bastante elevada, principalmente entre as capitais João Pessoa/PB e Natal/RN, que divergiram em $34,4 \%$ e as maiores cidades interioranas Campina Grande/PB e Mossoró/RN com uma divergência menor, de $10 \%$ entre a TI da COVID-19. As cidades de São Gonçalo do Amarante, Macaíba, Ceará-Mirim e Caicó não tiveram suas taxas reveladas no Painel COVID-19 do seu Estado ${ }^{(21)}$.

Quadro 4: Comparativo entre a Densidade Demográfica e a Taxa de Morbidade da COVID-19 na Paraíba e Santa Catarina e seus Municípios até 01 de Julho de 2020

COMPARATIVO ENTRE A DENSIDADE DEMOGRÁFICA E A TAXA DE MORBIDADE DA COVID-19 NA PARAÍBA E SANTA CATARINA E SEUS MUNICÍPIOS - 2020

\begin{tabular}{|c|c|c|c|c|c|}
\hline LOCAL & DM (HAB/KM2) & TM & LOCAL & DM (HAB/KM2) & TM \\
\hline Paraíba & 66,7 & $1,10 \%$ & Santa Catarina & 65,27 & $0,30 \%$ \\
\hline João Pessoa/PB & $3.421,28$ & $1,60 \%$ & Florianópolis/SC & 623,68 \\
\hline Campina Grande/PB & 648,31 & $1,60 \%$ & Joinville/SC & 457,58 & $0,30 \%$ \\
\hline Guarabira/PB & 333,8 & $3,80 \%$ & Blumenau/SC & 595,97 \\
\hline Cabedelo/PB & $1,815,57$ & $2,70 \%$ & São José/SC & $1.376,78$ & $0,50 \%$ \\
\hline Santa Rita/PB & 165,52 & $0,80 \%$ & Chapecó/SC & 293,15 \\
\hline Patos/PB & 212,82 & $1,30 \%$ & Itajaí/SC & 636,11 \\
\hline Mamanguape/Pb & 124,23 & $2,70 \%$ & Criciúma/SC & 815,87 \\
\hline (Fonte: IBGE, SES/PBE DEFESA CIVIL/SC, 2020) & & & $0,80 \%$ \\
\hline
\end{tabular}

Quadro 5: Comparativo entre a Densidade Demográfica e a Taxa de Incidência da COVID-19 na Paraíba e Rio Grande do Norte e seus Municípios até 01 de Julho de 2020

COMPARATIVO ENTRE A DENSIDADE DEMOGRÁFICA E A TAXA DE INCIDÊNCIA DA COVID-19 NA PARAÍBA E RIO GRANDE DO NORTE E SEUS MUNICÍPIOS - 2020

\begin{tabular}{|c|c|c|c|c|c|}
\hline LOCAL & DM(HAB/KM2) & TI & LOCAL & DM \\
\hline Paraíba & 66,7 & $30,3 \%$ & Rio Grande do Norte & 59,99 & $21,1 \%$ \\
\hline João Pessoa/PB & $3.421,28$ & $35,8 \%$ & Natal/RN & $4.805,24$ & $1,4 \%$ \\
\hline Campina Grande/PB & 648,31 & $11 \%$ & Mossoró/RN & 123,76 & $1,0 \%$ \\
\hline Guarabira/PB & 333,8 & $105,3 \%$ & Parnamirim/RN & $1.639,70$ & $1,2 \%$ \\
\hline Cabedelo/PB & $1.815,57$ & $48,71 \%$ & São Gonçalo do Amarante/RN & 351,91 & - \\
\hline Santa Rita/PB & 165,52 & $0 \%$ & Macaíba/RN & - \\
\hline Patos/PB & 212,82 & $0 \%$ & Ceará-Mirim/RN & 94,07 \\
\hline Mamanguape/PB & 124,23 & $127 \%$ & Caicó/RN & 51,04 \\
\hline (Fonte: IBGE, SES/PBE PAINEL COVID-19/RN, 2020) & & & - \\
\hline
\end{tabular}


Ainda levando-se em consideração a proporcionalidade da DM, foi possível correlacionar a TL da COVID-19 do Estado da Paraíba com o Estado do Ceará. Nesta comparação observou-se grande divergência, conforme pode ser observado no Quadro 6. O Estado do Ceará apresentou uma TL da COVID-19 de 5,7\%, durante o período estudado, mantendo uma TL, inclusive, mais elevada que a média nacional no mesmo período, que chegou a $4,0 \%$. Entre as capitais João Pessoa/PB e Fortaleza/CE a diferença entre a TL foi ainda mais notável, mais de $6,5 \%$ de divergência, o que pode ser atribuído a diferença da DM, tendo em vista que Fortaleza/CE possui uma DM maior que o dobro, quando comparado João Pessoa/PB.

Dentre os municípios do Estado do Ceará com menor TL, Crato/CE aponta $1,70 \%$, conforme Quadro 6, e possui uma DM de 103,21 hab/km2, essa mesma TL foi observada, durante o período deste estudo, na segunda maior cidade do Estado da Paraíba, Campina Grande/PB que possui uma DM cinco vezes maior, evidenciando que o Estado do Ceará foi realmente assolado pela pandemia ${ }^{(22)}$.

O Ceará registrou óbitos da COVID-19, durante o primeiro dia da doença até 01 de Julho de 2020, de in-

\section{O Estado do}

\section{Ceará apresentou}

uma TL da

COVID-19 de 5,7\%,

durante o período

estudado, mantendo

uma $T L$, inclusive,

mais elevada

que a média nacional

no mesmo período,

que chegou a $4,0 \%$. divíduos menores de um ano até 109 anos. Desses óbitos pela COVID-19 confirmados, $77,0 \%$ dos ocorreram com pessoas de idade entre 60 anos ou mais, com média de 72 anos, assemelhando-se com a faixa etária dos óbitos registrados na Paraíba. Em relação às comorbidades pré-existentes nos óbitos consecutivos de complicações da COVID-19, no Estado do Ceará prevaleceram as Doenças Cardiovasculares, Diabetes e Doença Renal Crônica, assemelhando-se às patologias apresentadas nos indivíduos que foram vítimas fatais da COVID-19 no território paraibano(22).

\section{CONCLUSÃO}

Diante dos resultados destacados no estudo, acerca da avaliação e descrição da distribuição espacial dos dados epidemiológicos dos casos da COVID-19 no Estado da Paraíba, pôde-se observar a contribuição das grandes cidades como fonte de propagação da doença, por sua alta DM, como também a similaridade dos dados encontrados na Paraíba com outros Estados do mesmo padrão de densidade e limites territoriais.

Pode-se perceber que a TI da COVID-19 apresentou-se mais elevada na capital paraibana, João Pessoa/PB, assim como o maior número de óbitos

Quadro 6: Comparativo entre a Densidade Demográfica e a Taxa de Letalidade da COVID-19 na Paraíba e Ceará e seus Municípios até 01 de Julho de 2020

COMPARATIVO ENTRE A DENSIDADE DEMOGRÁFICA E A TAXA DE LETALIDADE DA COVID-19 NA PARAÍBA E CEARÁ E SEUS MUNICÍPIOS - 2020

\begin{tabular}{|c|c|c|c|c|c|}
\hline LOCAL & DM (HAB/KM2) & $\mathrm{TL}$ & LOCAL & DM (HAB/KM2) & $\mathrm{TL}$ \\
\hline Paraíba & 66,7 & $2,08 \%$ & Ceará & 56,76 & $5,70 \%$ \\
\hline João Pessoa & $3.421,28$ & $2,70 \%$ & Fortaleza & $7.786,44$ & $9,40 \%$ \\
\hline Campina Grande & 648,31 & $1,70 \%$ & Caucaia & 264,91 & $7,80 \%$ \\
\hline Guarabira & 333,8 & $0,90 \%$ & Juazeiro do Norte & $1.004,45$ & $5,60 \%$ \\
\hline Cabedelo & $1.815,57$ & $1,00 \%$ & Maracanaú & $1.960,25$ & $6,00 \%$ \\
\hline Santa Rita & 165,52 & $6,70 \%$ & Sobral & 88,67 & $3,60 \%$ \\
\hline Patos & 212,82 & $3,80 \%$ & Crato & 103,21 & $1,70 \%$ \\
\hline Mamanguape & 124,23 & $1,30 \%$ & Itapipoca & 71,90 & $7,30 \%$ \\
\hline
\end{tabular}




\section{artigo}

Chagas, L.K.F.; Camargo, L.M.A.S.; Soares, L.T.B.; Lemos, L.F.; Dias, M.E.C.; Costa, R.A.;

Distribuição espacial da COVID-19 no estado da Paraiba: uma associação com a densidade demográfica

pela doença no Estado, durante o primeiro dia da doença até 01 de Julho de 2020. Apesar disso, João Pessoa/PB apresentou TL inferior em comparação à cidade de Santa Rita/PB, porém o limite territorial dessas cidades é comumente confundido, chegando o mu- nicípio de Santa Rita/PB a ser considerado grande João Pessoa/PB.

Compreendendo os padróes de disseminação relacionados com a quantidade de habitantes por $\mathrm{km}^{2}$, é possível auxiliar na adoção de medidas de saúde pública. Tendo os resultados do estudo como um legitimador da necessidade de manutenção das medidas preventivas, como é o caso do isolamento social, entre outra estratégias, a fim de garantir um baixo nível de contaminação e disseminação da doença, levando em consideração a existência de casos subnotificados e assintomáticos.

\section{REFERÊNCIAS}

1. Ministério da Saúde(BR). Coronavírus-COVID19. [Internet]. Brasília (DF): MS, 2020 [acesso em 23 jun 2020]. Disponível em: https://coronavirus.saude.gov.br/sobre-a-doenca\#o-que-e-covid.

2. Ministério da Saúde(BR). Coronavírus-COVID19. [Internet]. Brasília (DF): MS, 2020 [acesso em 23 jun 2020]. Disponível em: https://www.saude.gov.br/noticias/agencia-saude/46568-ministerio-da-saude-declara-transmissao-comunitaria-nacional.

3. Governo do Estado da Paraíba. Painel Coronavírus Paraíba. [Internet]. 2020 [acesso em 24 jun 2020]. Disponivel em: https:// paraiba.pb.gov.br/noticias/paraiba-confirma-primeiro-caso-de-coronavirus.

4. Carvalho Marilia Sá, Souza-Santos Reinaldo. Análise de dados espaciais em saúde pública: métodos, problemas, perspectivas. Cad. Saúde Pública [Internet]. 2005 [acesso em 24 jun 2020]; 21(2): 361-378. Disponivel em: https://doi.org/10.1590/S0102$311 \times 2005000200003$.

5. IBGE - Instituto Brasileiro de Geografia e Estatística(BR). [Internet]. Panorama Paraíba 2020 [acesso em 04 de jul 2020]. Disponivel em: https://cidades.ibge.gov.br/brasil/pb/panorama.

6. IBGE - Instituto Brasileiro de Geografia e Estatística(BR). [Internet]. Censo Demográfico João Pessoa 2020 [acesso em 04 de jul 2020]. Disponível em: https://cidades.ibge.gov.br/brasil/pb/ joao-pessoa/panorama.

7. IBGE - Instituto Brasileiro de Geografia e Estatística(BR). [Internet]. Censo Demográfico Campina Grande 2020. [ acesso em 04 de jul 2020]. Disponível em: https://cidades.ibge.gov.br/brasil/pb/campina-grande/panorama.

8. IBGE - Instituto Brasileiro de Geografia e Estatística(BR). [Internet]. Censo Demográfico Guarabira 2020 [acesso em: 04 de jul 2020]. Disponivel em: https://cidades.ibge.gov.br/brasil/pb/ guarabira/panorama.

9. IBGE - Instituto Brasileiro de Geografia e Estatística(BR). [Internet]. Censo Demográfico Cabedelo 2020 [acesso em 04 de jul 2020]. Disponível em: https://cidades.ibge.gov.br/brasil/pb/ cabedelo/panorama.

10. IBGE - Instituto Brasileiro de Geografia e Estatística(BR). [Internet].Censo Demográfico Santa Rita 2020. [acesso em: 04 de jul 2020] Disponivel em: https://cidades.ibge.gov.br/brasil/pb/ santa-rita/panorama.

11. IBGE - Instituto Brasileiro de Geografia e Estatística(BR). [Internet]. Censo Demográfico Patos 2020 [acesso em 04 de jul 2020]. Disponível em: https://cidades.ibge.gov.br/brasil/pb/patos/panorama.
12. IBGE - Instituto Brasileiro de Geografia e Estatística(BR). [Internet]. Censo Demográfico Mamanguape 2020 [acesso em: 04 de jul 2020]. Disponível em: https://cidades.ibge.gov.br/brasil/ pb/mamanguape/panorama.

13. IBGE - Instituto Brasileiro de Geografia e Estatística(BR). [Internet]. Censo Demográfico Joinville 2020. [acesso em 04 de jul 2020]. Disponivel em: https://cidades.ibge.gov.br/brasil/sc/joinville/panorama.

14. IBGE - Instituto Brasileiro de Geografia e Estatística(BR). [Internet].Censo Demográfico Florianópolis 2020 [acesso em 04 de jul 2020]. Disponível em: https://cidades.ibge.gov.br/brasil/sc/ florianopolis/panorama.

15. IBGE - Instituto Brasileiro de Geografia e Estatística(BR). [Internet]. Censo Demográfico Blumenau 2020 [acesso em 04 de jul 2020]. Disponível em: https://cidades.ibge.gov.br/brasil/sc/ blumenau/panorama.

16. IBGE - Instituto Brasileiro de Geografia e Estatística(BR). [Internet]. Censo Demográfico São José [acesso em 04 de jul 2020]. Disponivel em: https://cidades.ibge.gov.br/brasil/sc/sao-jose/ panorama.

17. IBGE - Instituto Brasileiro de Geografia e Estatística(BR). [Internet]. Censo Demográfico Itajaí 2020 [acesso em 04 de jul 2020]. Disponivel em: https://cidades.ibge.gov.br/brasil/sc/itajai/panorama.

18. IBGE - Instituto Brasileiro de Geografia e Estatística(BR). [Internet]. Censo Demográfico Criciúma 2020. [acesso em 04 de jul 2020]. Disponivel em:https://cidades.ibge.gov.br/brasil/sc/ criciuma/panorama.

19. IBGE - Instituto Brasileiro de Geografia e Estatística(BR). [Internet]. Censo Demográfico Chapecó 2020. [acesso em 04 de jul 2020]. Disponivel em:https://cidades.ibge.gov.br/brasil/sc/chapeco/panorama.

20. Governo de Santa Catarina. Plataforma multiescalar territorial COVID-19 [Internet]. 2020 [acesso em 03 jul 2020]. Disponível em: http://covid19.defesacivil.sc.gov.br/.

21. Universidade Federal do Rio Grande do Norte. Coronavírus RN [Internet]. 2020 [acesso em 03 jul 2020]. Disponivel em: https://covid.lais.ufrn.br/.

22. Ministério da Saúde(BR). Boletim Epidemiológico No 32 de 01 de Julho de 2020 [Internet]. 2020 [acesso em 03 jul 2020]. Disponível em :https://coronavirus.ceara.gov.br/project/boletim-epidemiologico-no-32-de-01-de-julho-de-2020/. 